\title{
Special conditions in the treatment of hemorrhoidal disease. The advantages of THD-RAR in the treatment of hemorrhoidal disease in these situations
}

\author{
Romica Mehic ${ }^{1}$, Veronica Indreica', Vasilica Marcu' ${ }^{2}$, \\ Mircea Beuran', Alexandru Laurentiu Chiotoroiu' ${ }^{1}$ \\ ${ }^{1}$ Emergency Clinical Hospital, Bucharest, Romania \\ ${ }^{2}$ Northampton General Hospital, Northampton, UK
}

\begin{abstract}
Introduction. The prevalence of hemorrhoids in the general population ranks first among benign anorectal diseases. In the last 20 years, however, the number of cases operated for hemorrhoidal disease has decreased and consequently the experience of surgeons in treating this condition has diminished and even less those cases associated with other complex pathologies that sometimes pose special treatment problems. Material and methods. We have studied the articles published in the period 1990-2020, which referred to special condition in patients with hemorrhoidal disease (young, elderly, pregnant woman, coagulation disorders, immunosuppressive states, colon inflammatory diseases, cirrhosis, renal failure, neurological diseases, associated perianal diseases). The recommendations we made were based on summing and updating the data found, conducting a critical review of the results of these studies, to which was added our personal experience, making special reference to the transanal hemorrhoidal dearterialisation (THD) technique.

Conclusions. The physiological features of the various stages of human evolution as well as the association with hemorrhoidal disease of other diseases that influence their symptoms and evolution require understanding in their close interdependence. Although hemorrhoidal disease is not a severe condition, it can affect the patient's social life or it can lead to unwanted complications or reinterventions if the therapeutic attitude is not adapted to the patient's associated conditions. The minimal damage of the anal anatomical structures in the THD technique makes the postoperative risks to be minimal even if a series of additional factors specific to the disease associated with hemorrhoids overlap. This makes THD a feasible therapeutic option for these at-risk patients.
\end{abstract}

Keywords: hemorrhoids, special conditions, treatment, THD

\begin{abstract}
Abbreviations
$\mathrm{RBL}=$ rubber band ligation

IS $=$ injection sclerotherapy

IRC $=$ infrared coagulation

$\mathrm{PPH}=$ procedure for prolapse and hemorrhoids
\end{abstract}

THD $=$ transanal hemorrhoidal dearterialisation THD-RAR = transanal hemorrhoidal doppler guided dearterialisation with/or without rectoanal repair or mucopexy

\section{INTRODUCTION}

The prevalence of hemorrhoids in the general population ranks first among benign anorectal diseases. Riss et al. [1] described a prevalence for hemorrhoids of $38.9 \%$ (patients diagnosed by colonoscopy and/or anoscopy). Only $44.7 \%$ of all these complained about symptoms associated with hem- orrhoidal disease, $27.1 \%$ had more severe forms of the disease (grade II-IV). 10\% of all symptomatic patients end up having surgery.

In the last 20 years, the number of cases operated on for hemorrhoidal disease has decreased, their place being taken by conservative and office treatments. As a result, the number of specialists who experience a wide range of surgeries for hemor- 
rhoids has decreased and even less so in cases associated with other complex pathologies that raise new question marks about the correct attitude.

Beyond the correct diagnosis of hemorrhoidal disease and its complications, it is important to identify the pathophysiological conditions and associated pathologies. We are often facing particular situations either in terms of the intervention of acute complications of hemorrhoidal disease (incarcerated hemorrhoidal prolapse, hemorrhoidal thrombosis), special physiological situations (particular attitudes in young people, the elderly or pregnant women) or the association of conditions which influence the course of hemorrhoidal disease (coagulation disorders, patients with immunosuppression, inflammatory colon disease, cirrhosis, chronic renal failure, neurological diseases, associated perianal diseases).

The purpose of this article is to put together data published in the literature related to all these special conditions together with our experience in the treatment of hemorrhoidal disease to help the young surgeon in understanding the clinical and evolutionary features of these situations and the best attitude he needs to have when they are in front of them, focusing on modern therapeutic trends in hemorrhoidal disease: office techniques (RBL $=$ rubber band ligation, IS = injection sclerotherapy, IRC = infrared coagulation), $\mathrm{PPH}$ (procedure for prolapse and hemorrhoids), THD-RAR (transanal hemorrhoidal doppler guided dearterialisation with/or without rectoanal repair or mucopexy).

\section{MATERIAL AND METHODS}

For preparing this article, we used data extracted from PubMed and Medline studying the articles published in the period 1990-2020, which referred to the topics that were discussed.

The recommendations or suggestions we made were based on summing and updating the data found in the extracted documents, conducting a critical review of the results of these studies, to which was added our personal experience.

\section{ACUTE COMPLICATIONS OF HEMORRHOIDAL DISEASE}

\section{Internal strangulated hemorrhoids}

It is a complication of internal hemorrhoids that after leaving the anal canal are not reduced. There is a disturbance in the vascular circulation, with edema, the progressive increase of volume that can lead, if not intervened in due time, to the necrosis of the mucosa. The simplest attitude is local anesthesia, with anal dilation and manual reduction, then the application of antiinflammatory ointments locally (a maneuver that sometimes needs to be repeated). There are opinions in the literature on the possibility of hemorrhoidectomy per primam taking care not to sacrifice too much mucosa [2]. From personal experience, this attitude can lead to delayed healing and stenosis. We prefer conservative treatment, followed by definitive treatment 30 days after the acute episode (THD-RAR or PPH).

\section{Hemorrhoidal thrombosis}

It is a complication due to the evacuation of a hard fecal bolus following constipation, which is associated with sustained skimming effort. It can also occur after intense physical exertion. Hemorrhoidal thrombosis is most commonly external, but can also be internal.

It is manifested by continuous, annoying pain in the anal region, which appeared on the background of palpation of a small paraanal swelling, like a pea of high consistency and purple color. Without surgery (local treatment with hemorrhoidal creams), the acute pain lasts 1-2 days, the symptoms disappearing completely after 2-3 weeks. It can leave behind an excess skin fold called a hemorrhoidal skin tag. Also, the frequency of relapses is higher after conservative treatment.

The operation is the option we support. It is done under in outpatient department, with local anesthesia, requires a minimal incision and leads to relief in a few hours of symptoms. Thrombectomy or excision of the entire hemorrhoid can be performed (the latter preferred by some authors with a lower risk of recurrence of bleeding or recurrence) [3]. From personal experience, we had no hemorrhagic accident and no recurrence after thrombectomy, using a small device in which we put a proximal hemostatic thread on the affected vessel, which also faces the edges of the wound.

\section{HEMORRHOIDS IN YOUNG PEOPLE}

The appearance of hemorrhoids in young people is a rarity. There are differences between children (1-9 years) and adolescents (10-19 years) regarding this disease.

Hemorrhoids in children are most often an epiphenomenon, secondary to another condition. We refer here to portal hypertension (the most common cause), colorectal neoplasm, rectal mucosal 
prolapse [4]. In conclusion, any child with hemorrhoids should also be investigated for the causal condition, especially for portal hypertension. The treatment of hemorrhoids in children is most often conservative (increased fiber intake, anti-itching creams, hemorrhoidal ointments) and only when the bleeding is persistent, minimally invasive interventions (rubber band ligation, injection sclerotherapy, infrared coagulation) are indicated.

Hemorrhoids in adolescents may be an independent disorder, but have a low incidence and self-limiting development. Consequently, conservative treatment is the first choice, as surgical treatment is often not necessary [5].

The main differential diagnosis is made with rectal prolapse, which requires a specific intervention, the prerogative of the pediatric surgeons. External trombosed hemorrhoids must also be distinguished from melanoma.

\section{HEMORRHOIDS IN THE ELDERLY}

We define the elderly patient a patient who is over 60 years old (WHO). The incidence of hemorrhoidal disease increases with age reaching $46.4 \%$ in the elderly which is one of the most common causes for which they refer to the doctor (besides proctitis, polyps, rectal cancer) [6]. 50\% of them receive treatment for hemorrhoidal disease, $10-20 \%$ requiring surgery.

Bleeding is the symptom that most frequently brings the patient to a doctor. A particular attention should be paid to the diagnosis in elderly patients because the cause of the bleeding may be often another than hemorrhoidal disease (diverticulosis, angiodysplasia, rectal ulcers, tumors), colonoscopy being indicated especially in cases where the origin of bleeding in the hemorrhoids is not obvious.

Regarding the particularities of these patients, in addition to constipation as a favoring factor, they are often treated chronically with antiplatelet which favors rectal bleeding as a symptom. Commonly they also have associated general conditions that confer an increased operative risk. Therefore, the decision regarding the operation of the elderly patient must be well weighed.

Particularly these patients may have a certain degree of impairment of the sphincter tone which must be carefully evaluated and specified preoperatively. They also exhibit greater tissue friability and a slower healing rate.

In the treatment of hemorrhoidal disease in the elderly we must pay special attention to the correction of constipation. We will choose minimally invasive procedures (rubber band ligation, sclerotherapy) and in cases with pronounced hemorrhoidal prolapse associated, interventions in which we should take care to preserve sphincterian function and as much anal mucosa as possible - THD-RAR, ligasure hemorrhoidectomy.

Taking into account these particularities, the surgical treatment of the elderly is equally as safe as in young patients with a complication rate comparable to the general population [7].

\section{HEMORRHOIDS IN PREGNANT WOMAN}

The presence of hemorrhoidal crisis is more common in pregnant women especially in the last trimester but also in the first 6 months postpartum. There are a number of factors that promote hemorrhoidal disease in pregnant women: 1 . increased circulatory volume; 2 . increased secretion of sex hormones (progesterone) that cause vascular congestion and in the same time induce constipation; 3 . increased abdominal pressure due to the pregnant uterus which grows congestion in the superior rectal plexus and in turn induces constipation; 4. the increased presence of constipation leading to an increased effort and the prolongation of the duration of defecation; 5. changes in the rectum induced during labor [8]. The frequency and intensity of the symptoms that the pregnant woman accuses are ameliorated and sometimes even disappear in six months from giving birth.

It should be noted that a number of medical treatments or therapeutic procedures are contraindicated in pregnant women due to fetal or maternal risk.

In general, surgical treatment should be postponed after birth especially since most of the symptoms improve or remit at 8-16 weeks, with disappearance of the causes that triggered them.

There are a number of measures that can counteract the effects induced by the physiological changes of the pregnant woman. Left lateral position and exercises helps lower pelvic pressure and improve circulation. Particular attention should be paid to the avoidance and treatment of constipation. Adequate water consumption especially in hot weather ( 8 glasses/day), adequate fiber consumption ( $30 \mathrm{~g} /$ day), use of volume laxatives - psyllium or methyl cellulose, osmotic laxatives - polyethylene glycol (10-20 g/day), lactulose (15-30 ml/day), stimulant laxatives (senna or bisacodyl) are all solutions available to the pregnant woman [9]. We must avoid emollient laxatives (oils) that reduce the absorption of vitamins and can induce premature 
contractions. Sitting baths and local treatments with cortisone can reduce itching and inflammation (the resorption in circulation is minimal). Also the use of flavonoids (Detralex) is allowed in the last quarter. In acute complications, the cases with severe bleeding or severe prolapse, or where conservative treatment fails, surgical intervention may be required: application of surgical or endoscopic elastic rings, injection sclerotherapy, infrared coagulation, hemorrhoidectomy by vascular sealing (diathermy, ligasure, harmonic scalpel) and lastly surgical hemorrhoidectomy can be done safely. Minimally invasive techniques are preferred, with local anesthesia and reduced hospitalization. THD-RAR can be an option especially after giving birth.

\section{HEMORRHOIDS IN PATIENTS WITH COAGULATION DISORDERS}

Sometimes we face the need to resolve complications of hemorrhoidal disease in patients with coagulation disorders either drug-induced (antiplatelets, anticoagulants) or accompanying other diseases that induce coagulation damage (hematological conditions, cirrhosis, chronic renal failure). In such cases, hemorrhoidal bleeding is often the main symptom and it is especially important as it is not self-limiting. Two essential questions must be asked: Does the patient take antiplatelet or anticoagulant treatment?; Is this patient known with any diseases that affect clotting?

\section{Drug-induced coagulation disorders}

Antiplatelet and anticoagulant medication is increasingly present in the chronic treatment of our patients, especially after 50 years. About one in five people receive chronic antiplatelet therapy, this proportion exceeding 50\% in the elderly. Antiplatelet agents (acetylsalicylic acid alone or in combination with P2Y12 receptor inhibitors such as Clopidogrel or Ticagrelor) are often used in acute coronary heart disease, stent implantation (for at least 6 weeks for BMS-type stents - metal stents and 12 months for DES-type stents - pharmacologically active stents) or stroke [10]. Oral anticoagulants (type of vitamin $\mathrm{K}$ antagonists Sintrom, Warfarine or new generation anticoagulants - Apixaban, Dabigatran, Rivarixaban) are indicated in atrial fibrillation, valve prostheses with metal valves, venous thromboembolism [10].

We will have to consider the severity of the hemorrhoidal disease, the type of treatment chosen, the severity of the condition for which anticoagulant or antiplatelet therapy is indicated, the risk of discontinuation of treatment. To adapt to the best attitude is required the collaboration between surgeon and cardiologist. The dilemma of therapeutic conduct lies in choosing the right type and time for surgery and assessment of the balance between the risk of bleeding and the risk of complications after discontinuation of anticoagulant/antiplatelet therapy. All of these medicines can precipitate bleeding events, but the risk of bleeding is generally lower than the risk of thrombosis. From the point of view of type of surgery, rubber band ligation, $\mathrm{PPH}$ and open hemorrhoidectomy are considered interventions with the highest risk of bleeding. At the opposite pole, infrared coagulation, injection sclerotherapy or THD are considered interventions with a low risk of bleeding [13].In terms of thrombotic risk, it can be assessed by measuring the CHADS-VASc score (who follows the presence of heart failure, hypertension, age, diabetes, history of stroke or thromboembolism, sex). The risk of bleeding is assessed by the HAS-BLED score (following the presence of hypertension, renal or hepatic impairment, bleeding history, INR lability, age over 65 years, consumption of drugs that may influence clotting or alcohol) $[11,13]$. Depending on the value of these scores, the patient falls into low, medium or high risk groups according to which it is decided to discontinue the anticoagulant medication, switch to bridge therapy with drugs whose effect is short and whose doses are easy to control (heparin, low molecular weight heparins), postponing surgery or choosing procedures with a low risk of bleeding when discontinuation of the antiplatelet/anticoagulant has too high a risk of complications.

\section{Diseases that affect coagulation}

These are another situation that we need to keep in mind. Hemophilia A (recessive hereditary condition that mainly affects males and is characterized by quantitatively diminished or qualitatively altered synthesis of coagulation factor VIII) is a particular case of coagulation disorder. In these cases, any surgery must be thoroughly prepared in collaboration with the hematologist by: assessing preoperative coagulability (the presence of more than $25 \%$ of the normal percentage of factor VIII ensures effective hemostasis), preoperative administration of cryoprecipitate or factor VIII concentrate (IX), the choice of a minimally invasive procedure (sclerosing injections or THD) with the avoidance of hemorrhoidectomy, careful control of 
hemostasis at the end of the intervention. Postoperative development of factor VIII inhibitors may be the cause of persistent bleeding requiring reintervention to control hemostasis [14]. Bleeding morbidity reaches rates of $6-23 \%$, usually occurring from the first day postoperatively (with a maximum on day VI), while mortality tends to zero after the introduction of factor VIII and IX concentrates.

\section{HEMORRHOIDS IN IMMUNOCOMPROMISED PATIENTS}

Patients with autoimmune deficiencies (leukemia, lymphoma, HIV positive or AIDS, immunotherapy) generally have higher risk of infections, coagulation disorder due to thrombocytopenia, slowing the wound healing process. The frequency of hemorrhoid bleeding is higher in these patients. We need to consider in HIV patients the possibility that they may have a series of associated anno-rectal disorders: fissures or perianal fistulas, venereal lesions (condylomas, syphilis, herpetic ulcerations), malignant lesions (Kaposi sarcom, anal epidermoid cancer) [15]. Hemorrhoidectomy has to be avoided in patients with AIDS or other autoimmune diseases. They have a high complication rate $(87.5 \%$ in patients with AIDS, respectively $22 \%$ in HIV positive patients) and a high rate of slow healing (only $50 \%$ cures at 32 weeks in patients with AIDS) [17]. Surgical treatment of hemorrhoids in HIV patients should be reserved for those in whom medical treatment is not effective or with hemorrhoid prolapse. Coagulation disorders should be corrected and antiviral treatment (Tenefovir, Lamivudine, Efavirenz) should be given in patients with CD 4 below 350/microl. Preoperative local toilet and antibiotic treatment are of particular importance in these patients. Minimally invasive procedures by rubber band ligation or injection sclerotherapy can be considered safe and effective in asymptomatic HIV positive patients. Good results have also been reported by tissue selective stepler (TST) [16].

\section{HEMORRHOIDS IN INFLAMMATORY BOWEL DISEASES}

Inflammatory colon diseases (Crohn's disease and ulcerative colitis) pose special problems of diagnosis but also of choosing the indication and type of surgery given the high risk of postoperative complications. Repeated inflammatory attacks and local irritation caused by diarrhea may precipitate the development of hemorrhoidal attackes.
In Crohn disease, hemorrhoids can be confused with hypertrophic papillae (which are pink not purple, more sensitive and often associated with ulcerations of the anal canal). It is important to appreciate if the patient has inflammatory bowell disease (rectal ulceration, diarrhea stools), if this disease is active and how severe it is (according to the Crohn disease activity index, C-reactive protein level, length of the affected intestine). $20-36 \%$ of perianal disorders are preceded by inflammatory colonic disorders. The incidence of hemorrhoidal disease appears lower in patients with Crohn disease (7\%) than in general population (24\%). The general principle is to avoid surgery on patients known to have Crohn disease due to the high rate of complications (delay in wound healing, bleeding, fistulas, sepsis, stenosis). Conservative medical treatment is especially recommended in patients with inflammatory bleeding. A surgical technique may be indicated in these patients if the inflammatory disease is stable, they are not under steroid treatment and have an activity index of less than 150. PPH technique is not recommended. However, the application of rings, THD-RAR, hemorrhoidectomy (in exceptional cases) have obtained acceptable results. The number of complications was $41.2 \%$ (compared to $15-25 \%$ in the general population). It is important to mention that complications were significantly rare if the diagnosis of Crohn disease was made before the intervention for hemorrhoids (due to a correct treatment of inflammatory disease and the choice of a more conservative procedure for the treatment of hemorrhoidal disease).

In ulcerative colitis the hemorrhoids reach a cumulative incidence at 10 years of $6.7 \%$ at a total incidence of perianal diseases of $16 \%$ [16]. Although it does not pose such delicate postoperative evolution problems as Crohn disease, neglecting the diagnosis, acute outbreaks or influences of the specific treatment can lead to undesirable therapeutic failures. Proper targeted history and indication when needing recto-colonoscopy with careful follow up of rectal mucosal appearance and biopsy result will help to make preoperative diagnosis (diarrhea stools with blood and pus more than 4 weeks, ulcerative lesions with continuous appearance and endoscopic circumference, chronic inflammation in the lamina propria with distortions of the crypts and ulcers on histopathological examination). On the other hand, careful anoscopy, carried out by an experienced proctologist, can keep us out of the wrong interpretation of a hemorrhoid bleeding or the presence of a fissure as an inflammatory burst of the ulcerative colitis and as a result of escalating 
unnecessary stress on relapse of the disease and of inadequate therapy. Male sex and the extent of inflammatory disease were found to be statistically significant risk factors, also contributing the age below 40, smoking, immune treatment. Bleeding and pain are the main symptoms. $42.1 \%$ responded favorably to conservative treatment [16]. Nonexcisional techniques (rubber ban ligation, injection sclerotherapy, THD) were also preferred for those who received surgical treatment.

\section{HEMORRHOIDS IN HEPATIC CIRRHOSIS}

Portal hypertension has the most common cause liver cirrhosis. In portal hypertension, venous dilatations occur at the level of certain communication areas between the portal venous system and the vena cava system, these being called varicose veins, the erosion of which can cause bleeding. Varicose veins occur most frequently in the esophagus but they can also occur in other levels of the digestive tract such as the rectum.

In patients with portal hypertension we can observe anorectal varicose veins (33\% of cases), hemorrhoids $(35 \%)$ or external anal varicose veins (15\%). Rabinovitz et al. [18] found a prevalence for rectal varicose veins of $3.6 \%$, respectively for hemorrhoids of $25.2 \%$ on a group of 412 patients with liver cirrhosis. Hemorrhoids can coexist with rectal varicose veins. However, the frequency of hemorrhoids in patients with portal hypertension does not differ from the general population, instead the frequency of rectal varicose veins is directly proportional to the values of portal hypertension and the severity of cirrhosis. Varicose veins occur at values greater than $12 \mathrm{mmHg}$ of portal hypertension (nor$\mathrm{mal}=1-5 \mathrm{mmHg}$ ).

The particularities of cirrhotic patients are that hemorrhoids can be confused or coexist with rectal varicose veins, these patients sometimes have severe haemostasis due to decreased hepatic coagulation factors and thrombocytopenia secondary to hypersplenism, may have decompensation of liver function (ascites, encephalopathy) and have an increased anesthetic risk.

Hemorrhoids differ from anorectal varicose veins by pathophysiology, location, complications and last but not least by treatment.

The symptom for which the patient most often presents is bleeding. It is important to know the source of the bleeding, because the treatment of hemorrhoidal bleeding differs from the treatment of rectal variceal bleeding. Hemorrhoid bleeding is usually red-blooded, is quantitatively lower, and self-limiting, while varicose veins are darker red, more pronounced, and tend to persist. Hemorrhoids are located immediately above the anocutaneous line with which they are in continuity and are systematized in three areas (middle left, right anterior and right posterior in gynecological position), varicose veins are located higher, in the lower rectum, having an area of separation from the anocutaneous line. For the diagnosis of hemorrhoids, anoscopy performed by a specialist is sufficient, while for varicose veins, endoscopy, endoscopic ultrasonography, Doppler ultrasound of the port system are required.

In the treatment of rectal varicose veins we must consider both hemostasis and correction of portal hypertension. For hemostasis the endoscopic methods are of first choice by sclerosing injections, the application of endoscopic elastic rings, the intravariceal injection of cyanoacrylate, when the endoscopic methods fail being able to resort to simple ligature surgical methods, surjet on the bleeding vessel, stapling. For the correction of portal hypertension, transjugular intrahepatic porto-systemic shunt can be used, embolization of the veins that supply the rectal varicose veins (a method that also has a direct hemostatic effect), and a surgical porto-caval shunt can be performed surgically [19]. Without the combination of these measures, bleeding is often difficult to control or recur.

In the treatment of hemorrhoids, miniinvasive techniques are preferable due to the increased anesthetic and surgical risk. The application of elastic rings is to be avoided due to the risk of postoperative bleeding in these patients. Miyamoto $\mathrm{H}$. et al. obtains encouraging results using sclerosing injections with aluminum potassium sulfate and tannic acid (ALTA), using for identification of blood flow in hemorrhoids and rectal varicose veins power-Doppler three-dimensional angiography [20]. Galati G. et al. publishes a case of hemorrhoids in which he practices TIPS in which due to the persistence of bleeding he performs THD with good results at 15 months [21]. Elsebae M.M. et al. publish the results of a group of 30 patients operated on for hemorrhoidopexy by stapling with a percentage of $46.7 \%$ (14 p) of minor bleeding from the stapling line, which did not require reintervention and disappeared completely after three months [22].

\section{HEMORRHOIDS IN CHRONIC RENAL INSUFFICIENCY}

The modernization and the funds allocated to the departments of chronic dialysis or peritoneal di- 
alysis have lately increased the life expectancy of dialysis patients and consequently increased the share of patients with renal insufficiency among those who need surgery. Sheikh et al. [23] had a percentage of $8.37 \%$ patients who required anorectal intervention, the majority $(81.8 \%)$ being for hemorrhoidal disease.

Chronic renal failure is a particular feature due to disorders in protein metabolism that lead to increased friability of the skin and mucous membranes to these patients and the frequent association of anemia. Anemia may be due to decreased erythropoietin production and decreased survival of red blood cells or digestive losses. These patients additionally have coagulation disorders induced by haematological defects and treatment with heparin (given during hemo-dialysis) or with anti-aggregates. In peritoneal dialysis the additional pressure caused by dialysis fluid is an additional factor that induces constipation and hemorrhoidal disease.

The presence of blood in the stool may be the source of the upper digestive tract in $10 \%$ of cases, intestine $5 \%$ or colon and rectum $85 \%$. Although most of the bleeding stops spontaneously, they must be investigated because the bleeding recurs in a proportion of $40 \%$ the more as they appear on the field of pre-existing anemia. Hemorrhagic disease ranks $3^{\text {rd }}(14 \%)$ as a cause of lower digestive haemorrhages after angiodysplasia, diverticulosis and later come the anal fissure, stercoral ulcerations [24].

Conservative treatment is the first choice in hemorrhoidal disease with measures to reduce constipation, increase fiber consumption, laxatives but the consumption of water has to be adapted to diuresis (24 hour urine excreted plus $500 \mathrm{ml}$ ). Patients with renal failure and hemorrhoidal disease generally come for bleeding, Hemorrhoid banding is the safest and most effective method. Sclerotherapy, infrared coagulation, THD may be other therapeutic options. Hemorrhoidectomy is preventable due to mucosal friability in these patients and the risk of postoperative bleeding.

\section{HEMORRHOIDS IN NEUROLOGICAL DISEASES}

Neurological disorders and especially those that affect to an advanced extent the spinal cord function (traumatic spinal cord injuries, degenerative diseases, genetic diseases - Charcot-Marie-Tooth) are often accompanied by disorders involving the digestive tract. Disorders of the lower digestive tract take first place in this context. Constipation is characteristic for these patients as well as its secondary diseases: hemorrhoids, stercoral ulcers, mega-bowell, bowell volvus. The prevalence of hemorrhoids in these patients is much higher than in the general population $42-81 \%$ [25]. Often these patients appeal to auxililiary maneuvers to resolve constipation (enema, manual extraction of fecalomas) which favors inflammation or damage to hemorrhoids. In this context and due to the persistence of the favoring factors for the progression of the hemorrhoidal disease (constipation, repeated mechanical trauma) the minimally invasive surgical techniques seem the most appropriate (PPH or THD + RAR) and less the resection ones (hemorrhoidectomy) being the only ones that can give good result in long term especially because in these patients prolapse is the dominant symptom and incontinence of different degrees is often associated with hemorrhoidal pathology.

\section{HEMORRHOIDS AND ASSOCIATED PERIANAL DISEASE}

Besides hemorrhoids there are also others anorectal conditions who can compete for the symptomatology for which the patient is presented to the doctor, being able to give specific symptoms at the same time. In case of presence of a condition associated with hemorrhoids, the attending physician is facing the situation to decide which is the most appropriate therapeutic attitude. Generally, the condition that most endangers the patient's life is the one that prevails in dictating therapy.

First of all, the doctor must use all the arsenal at his disposal for a correct diagnosis (anamnesis, inspection of the anal region, rectal tacsis, anoscopy, colonoscopy, biopsy, exploratory puncture). Within the associated diseases we can meet with: hemorrhoidal, anal polyp, anal fissure, perianal suppurations (absces or fistula), mucosal ectropion, mucous or complete rectal prolapse, rectocele, rectal polyp, ano-rectal cancer [3].

The presence of hemorrhoidal skin tags, of an anal hyprtrophic papilla or of an anal polyp can be treated at the same time with hemorrhoids: either by an excisional method that targets both the hemorrhoids and the polyp or by a minimally invasive method for internal hemorrhoids and the excision of the skin tags or polyp.

The mucosal ectropion is a common consequence of Whitehead circumferential hemorrhoidectomy. Hemorrhoidal recurrence in case of mucosal ectropion should benefit from a minimally invasive intervention (THD, rubber band ligations, 
injection sclerotherapy) given the anatomy already modified by the previous intervention.

Hemorrhoids associated with rectal mucosal prolapse can be treated concomitantly by stapling hemorrhoidectomy.

The perianal fissure may benefit from concomitant treatment with hemorrhoids, the anal sphincter relaxation intervention (botulinum toxin injection or partial internal sphincterotomy) having in turn a pathophysiological role in the healing of hemorrhoids.

In case of perianal suppurations (abscess, fistula) their treatment takes precedence followed by the treatment of hemorrhoids in the second time using minimally invasive methods (rubber band ligation, THD).

Detection of a tumor when exploring the anal region requires a diagnostic approach (biopsy) and therapeutic (excision, radiotherapy, resection) first of the neoplasia.

\section{THE ROLE OF THD (TRANSANAL HEMORRHOIDAL DEARTERIALISATION) AND RAR (RECTO-ANAL REPAIR) IN THE TREATMENT OF SPECIAL SITUATION FOUND IN HEMORRHOIDAL DISEASE}

THD is a mini-invasive, non-excisional, modern surgical technique used on an increasing scale in the treatment of hemorrhoidal disease wich has as principle the decrease of the blood supply in hemorrhoidal plexus by ligating the branches of the superior rectal artery after identifying them with the Doppler probe. RAR is an associated procedure introduced later in the surgical practise wich improves the results of THD in hemorrhoidal disease with prolapse in varying degrees by applying a continuous suture of hemorrhoidal mucosa with prolapse that will lead to the reintegration of the mucosa in a normal anatomical position.

Our experience using the THD-RAR technique in special conditions is encouraging. We must pay atention to the particularities of the associated diseases in order to correct possible deficits (the anemia, the coagulation disorders, the acute episodes) and to choose the right moment for the surgical treatment. We keep in mind also that for some situation the treatment is still in the evaluation phase.

Performing THD-RAR technique in acute complications of hemorrhoidal disease (hemorrhoidal thrombosis, incarcerated hemorrhoids) is not indicated. In these cases a medical treatment and possibly surgery after 30 days are preferred.
For elderly patients, the operation is indicated in cases of bleeding or prolapse, except for severe anal stenosis or incontinence.

In pregnancy, conservative treatment is preferred, while the surgery is generally performed after the third month.

For patients with coagulation disorders, THD-RAR technique is preferred to hemorrhoidectomy due to decreased risck of bleeding.

For the cases with inflammatory bowel disease, THD involves a minimal aggression on the mucosa and is preferred to other excisional techniques. For Crohn's disease patients, an asymptomatic period of disease is required in order to operate.

For portal hypertension cases, the surgery should be avoided but if the bleeding can't be stopped otherwise then the continuous suture from THD-RAR technique can be used with good outcome especially for the patients with varicose veins. We need to think about correction of coagulation disorders and the thrombocytopenia.

Patients with renal impairment may be operated with THD-RAR technique in good condition but we need to be careful at anuscope handling to not cause mucosal damage and at the end of the procedure to control hemostasis.

In Surgery Departament of the Emergency Hospital Bucharest, we had 9 cases operated with THD-RAR technique with special pathologies associated, representing $18 \%$ of the cases operated by this method: 2 cases of incarcerated hemorrhoids with mucosal necrosis, 2 cases of hemorrhoids during pregnancy operated after birth, 3 cases with renal impairment and 2 cases with neurological impairment from Charcot-Marie-Tooth disease. As incidents, we had a lesion of rectal mucosa by handling the anuscope at one of the patients with renal impairment and one lesion by inserting the hemostatic sponge, both resolved by passing an additional hemostatic thread. As early complications, we had a bleeding 5 days postoperatively at one of the patients with kidney failure for whom an elastic ring was applied. Three patients relapsed, two of them with prolapse for wich a reintervention THD-RAR was made with good outcome in one case and for the other one hemi-Whitehead hemorrhoidectomy was needed and one case of bleeding 2 years after surgery for which elastic rings were applied.

\section{CONCLUSIONS}

It is clear that the physiological particularities of the various stages of human life as well as the 
association with hemorrhoidal disease of other diseases that influence the symptoms and their evolution require understanding in their close interdependence.

In some cases, it is necessary to treat the associated condition simultaneously (coagulation disorders, immune disorders, inflammatory colon diseases), in others it is necessary to adapt the hemorrhoidal therapeutic technique to the particularities induced by the associated disease (old age, pregnancy, associated perianal diseases, cirrhosis, kidney failure).

Although hemorrhoidal disease is not a severe condition, it can affect the patient's social life and can lead to unwanted complications or reoperations if the therapeutic attitude is not adapted to the patient's associated conditions.

\section{REFERENCES}

1. Riss S, Weiser FA, Schwameis K, Riss T, Mittlbock M, Steiner G, et al. The prevalence of hemorrhoids in adults. Int J Colorectal Dis. 2012; 27:215-20.

2. Ackland TH. The treatment of prolapsed gangrenous haemorrhoids. Aust NZJ Surg. 1961;30:201-203.

3. Yang HK. Hemorrhoids in a Specific Condition. in Hemorrhoids. Springer-Verlag, Berlin Heidelberg, 2014:26-123.

4. Gupta PJ. Grade III bleeding haemorrhoids in a 6-year-old boy. Surgical Practice. 2005;9:50-52.

5. Karavelioglu AK, Senaili A, Koseoglu B, Akin M, Ozguner IF. Haemorrhoids in Children: A Retrospective Study. J Contemp Med. 2015;5(2):106-110.

6. Kowaska WL, Poniewierka E, Sapian B. Proctologic diseases in geriatric persons. Materia Medica Polona 1991;23(1):29-32.

7. Haksal MC, Yazicioglu MB, Tiryaki C, Ciftci A, Coskun M, Yildiz SY. Safety of surgical treatment of hemorrhoids in elderly patients. J Health Sciences. 2015;5(3):99-103.

8. Parangi S, Pories S. Surgical problems in the pregnant patient. Pregnancy in Gastrointestinal Disorders, 2nd ed. American College of Gastroenterology, 2007:54-64.

9. Christie JA, Rose S. Constipation, Diarrhea, Hemorrhoids and Fecal Incontinence. Pregnancy in Gastrointestinal Disorders, 2nd ed. American College of Gastroenterology, 2007:4-9.

10. McBeth PB, Weinberg JA, Sarani B, et al. A surgeon's guide to anticoagulant and antiplatelet medications part one: warfarin and new direct oral anticoagulant medications. Trauma Surg Acute Care Open. 2016;1:1-5.

11. Yeung LYY, Sarani B, Weinberg JA, McBeth PB, May AK. Surgeon's guide to anticoagulant and antiplatelet medications part two: antiplatelet agents and perioperative management of long-term anticoagulation. Trauma Surg Acute Care Open. 2016;1: 1-7.

12. Atallah S, Maharaja GK, Martin-Perez B, Burke JP, Albert MR, Larach SW. Transanal hemorrhoidal dearterialization (THD): a safe procedure for the anticoagulated patients? Tech Coloproctol. 2016;20:461-466.

13. Albright J, Bailey R. Hemorrhoid therapy for patients on medications altering coagulation. In Khubchandani I, Paonessa N, Khawaja A, eds.
THD-RAR is a modern method, with a proven efficacy for hemorrhoidal bleeding and stage II-III hemorrhoidal prolapse and which by minimal damage to the mucosa, minimal direct vascular injuries and lack of leaving bare areas can be a minimally invasive surgical solution in patients at increased associated risk: elderly, associated coagulation disorders, immunosuppression, inflammatory diseases, friability or poor scarring of the rectal mucosa, situations approaching $20 \%$.

Knowing all these situations, their peculiarities, the influence they sometimes have on the evolution of hemorrhoidal disease, is important in taking the best decision on the treatment of hemorrhoidal disease.

Conflict of interest: none declared Financial support: none declared

Surgical treatment of hemorrhoids. 2nd ed. Springer-Verlag, London, 2009:165-174.

14. Guideline for the management of patients with haemophilia undergoing surgical procedures. Australian Haemophilia Centre Directors Organisation. Nov 2010.

15. Gecim IE. Management of Hemorrhoidal Disease in Special Condition. In Ratto $\mathrm{C}$ et al. Hemorrhoids, Coloproctology 2. Springer International Publishing AG. 2018:113-118.

16. Fan Z, Zhang Y. Treatment of prolapsing hemorrhoids in HIV-Infected patients with tissue-selecting technique. Gastroenterol Research and Practice. 2017;1:5

17. Hewitt WR, Sokol TP, Fleshner PR. Should HIV status alter indications for hemorrhoidectomy? Dis Colon Rectum. 1996;39:615-618.

18. Rabinovitz M, Schade RR, Dindzans VJ et al. Colonic disease in Cirrhosis. An endoscopic evaluation in 412 patients. Gastroenterology. 1990;99:195-199.

19. Miyamoto H, Nakagawa T, Miyamoto Hideyuki, Takata A. Sclerotherapy using aluminum potassium sulfate and tannic acid (ALTA) for haemorrhoids in patients with liver cirrhosis. Ann Colorectal Res. 2015;3(4):e32980.

20. Sato T. Treatments for rectal varices with portal hypertension. J Gastroint Dig Syst. 2013;S6:1-2.

21. Khalloufi KA, Laiyemo AO. Management of rectal varices in portal hypertension. World J Hepatol. 2015;7(30):2992-2998.

22. Weinshel E, Chen W, Falkenstein DB, Kessler R, Raicht RF. Hemorrhoids or rectal varices: Defining the cause of massive rectal hemorrhage in patients with portal hypertension. Gastroenterol. 1986;90:744-7.

23. Sheikh F, Khubchandani IT, Rosen L, Sheets JA, Stasik JJ. Is anorectal surgery on chronic dialysis patients risky? Anorectal Surgery. 1990;35(1):56-57.

24. Saeed F, Agrawal N, Greenberg E, Holley JL. Lower gastrointestinal bleeding in chronic hemodialysis patients. International Journal of Nefrology. 2011;Article ID 272535:8 pages.

25. Ebert E. Gastrointestinal involvement in Spinal Cord Injury: a Clinical Perspective. J Gastrointestin Liver Dis. 2012;21(1):75-82. 